\title{
Eidechsen als Nektartrinker - erste Beobachtungen auf dem afrikanischen Festland
}

Petra Wester

\begin{abstract}
In South Africa, pollination by vertebrates is known for birds and small mammals. During video surveillance of plants adapted to small mammal pollination, lizards were observed at the Pineapple lily, Eucomis regia, and Massonia grandiflora (both Asparagaceae). The Cape cliff lizard (Hemicordylus capensis, Cordylidae) and the Namaqua day gecko (Rhoptropella ocellata, Gekkonidae) licked nectar from the flowers, touching the flowers' reproductive organs and getting dusted with pollen on their head. Thus, it is very likely that the lizards contribute to pollination. Pollination by lizards is a rare phenomenon and is largely restricted to islands. These are the first observations of lizards drinking floral nectar on the African mainland.
\end{abstract}

\section{Zusammenfassung}

Als Wirbeltiere, die Blüten bestäuben, kennt man in Südafrika Vögel und Kleinsäuger. Beim Video-Monitoring von Pflanzen, die an bestäubende Kleinsäuger angepasst sind, wurden Eidechsen an der Ananaslilie (Eucomis regia) und an Massonia grandiflora (beides Asparagaceae) beobachtet. Der Kap-Gürtelschweif (Hemicordylus capensis, Cordylidae) und der Namaqua-Taggecko (Rhoptropella ocellata, Gekkonidae) leckten Nektar, berührten die Reproduktionsorgane der Blüten und wurden dabei mit Pollen auf dem Kopf eingestäubt. Folglich ist es sehr wahrscheinlich, dass die Eidechsen zur Bestäubung der Blüten beitragen. Blütenbestäubung durch Eidechsen ist ein sehr seltenes Phänomen und ist weitgehend auf Inseln beschränkt. Dies sind die ersten Beobachtungen von nektartrinkenden Eidechsen auf dem afrikanischen Festland.

\section{Eidechsen als Überraschungsgäste an Blüten kleinsäugerbestäubter Pflanzen}

Der Prozess der Bestäubung von Blüten ist eine der faszinierendsten Interaktionen zwischen Pflanzen und Tieren. Tiere, die Blüten bestäuben, sind meist Insekten (vor allem Bienen), aber auch Wirbeltiere. Im Gegensatz zu den gut untersuchten blütenbestäubenden Vögeln und Fledermäusen sind nichtfliegende Kleinsäuger als Bestäuber selten und relativ unbekannt. In Südafrika sind Nagetiere wie Mäuse, aber auch Vertreter der Afrotheria, die Rüsselspringer, regelmäßige Blütenbesucher und Bestäuber von bestimmten Pflanzenarten (Wester 2009, 2011). Die Blüten dieser Pflanzen zeigen Merkmale, die wahrscheinlich als Anpassung an diese bestäubenden Kleinsäuger entstanden sind.

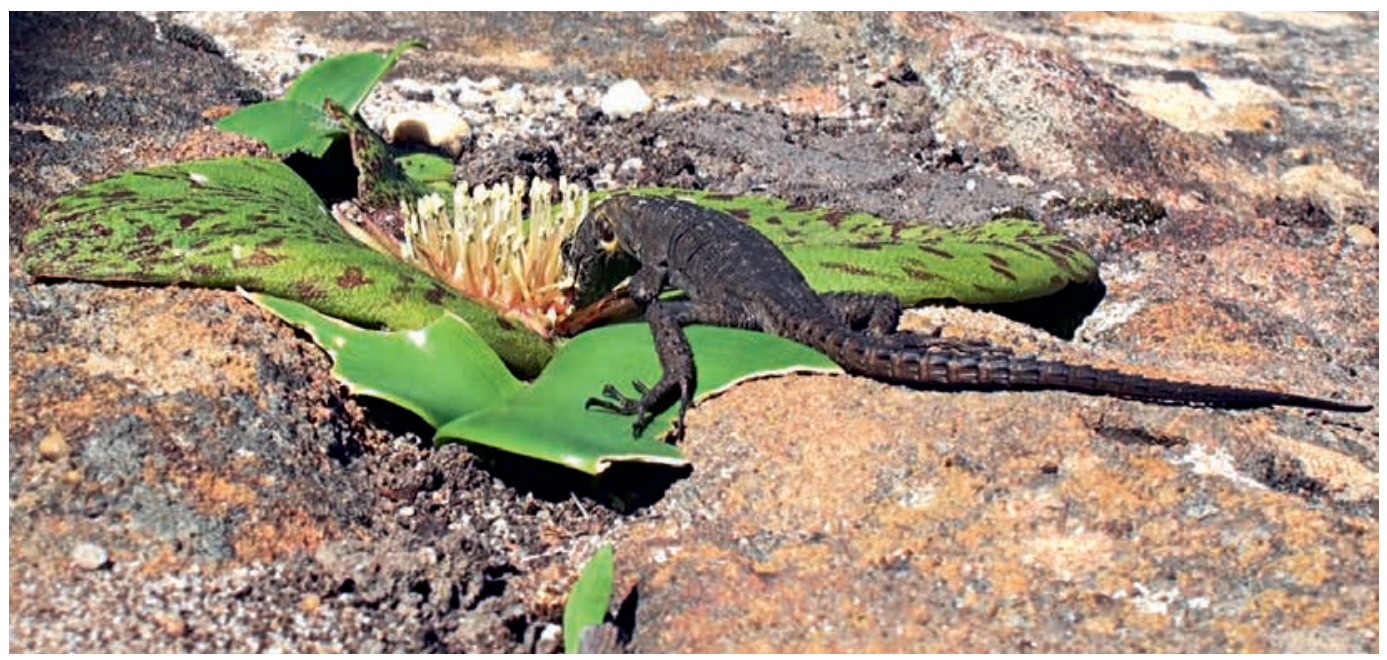

Abb. 1: Ein Kap-Gürtelschweif (Hemicordylus capensis) trinkt Nektar an Blüten von Massonia grandiflora in den Cederbergen Südafrikas. Man sieht die Antheren, die die Schnauze der Eidechse mit Pollen einstäuben. (Foto: aus Videomaterial von P. Wester) 
Dazu gehören z. B. Geoflorie (Blüten am Boden), visuelle Unauffälligkeit sowie robuste, napfförmige Blüten mit leicht erreichbarem Nektar und einem spezifischen Duft. Zwei Vertreter dieser Pflanzen, die Ananaslilie (Eucomis regia) und Massonia grandiflora (beides Asparagaceae), werden von Mäusen und Rüsselspringern bestäubt (Wester et al. 2019, Wester, unveröffentlichte Daten). Beim Monitoring dieser Pflanzen mit Videokameras und Kamerafallen (einige Tage in den Jahren 2014, 2016 und 2017) in den Cederbergen und im Namaqualand Südafrikas, wurden neben Kleinsäugern überraschenderweise auch Eidechsen an den Blüten beobachtet.

\section{Nektartrinkende Eidechsen als potenzielle Bestäuber}

An fünf Tagen konnte der Kap-Gürtelschweif (Hemicordylus capensis) beim Besuch von drei verschiedenen Massonia grandiflora-Pflanzen beobachtet werden. Zwei Ananaslilien wurden an einem Tag vom Namaqua-Taggecko (Rhoptropella ocellata) besucht. Beide Eidechsenarten besuchten die Pflanzen tagsüber. Sie liefen auf die Blütenstände zu und schoben ihren Kopf in die Blüten. Es war eindeutig zu sehen, dass die Echsen Nektar leckten und dabei die Pollensäcke der Blüten berührten. Das Berühren der Narben der Blüten war schwieriger zu beobachten, aber einige Male erkennbar. Beim Kap-Gürtelschweif konnte man den Pollentransfer auf seinen Kopf klar beobachten. Aufgrund des Punktemusters der Echsen (weiß auf schwarz) war die Pollenablage auf den Kopf der Namaqua-Taggeckos nur sehr schwer zu erkennen, was aber möglicherweise einige Male auftrat.

Diese Beobachtungen demonstrieren zweifellos, dass zwei Echsenarten zielgerichtet die Blüten der beiden Kleinsäuger-bestäubten südafrikanischen Pflanzenarten besuchen. Während Blütenbesuche des Namaqua-Taggeckos nur an einem Tag (von zwei Beobachtungstagen) beobachtet wurden, wurde der Kap-Gürtelschweif an fünf Tagen in zwei aufeinanderfolgenden Jahren registriert. Dies ist bedeutsam, da sogar die Hauptbestäuber Blüten nicht unbedingt so regelmäßig besuchen. Dies zeigt, dass das Verhalten der Eidechsen keine Ausnahmeerscheinung ist. Das Nektartrinken der Echsen war eindeutig erkennbar, vor allem beim Kap-Gürtelschweif an den besser erkennbaren Massonia-Blüten. Selten (oder nie beim Namaqua-Taggecko) fingen die Eidechsen Insekten. Da die mit Pollen bedeckten Kopfbereiche der Tiere mit den Kopfbereichen überlappten, die mit den

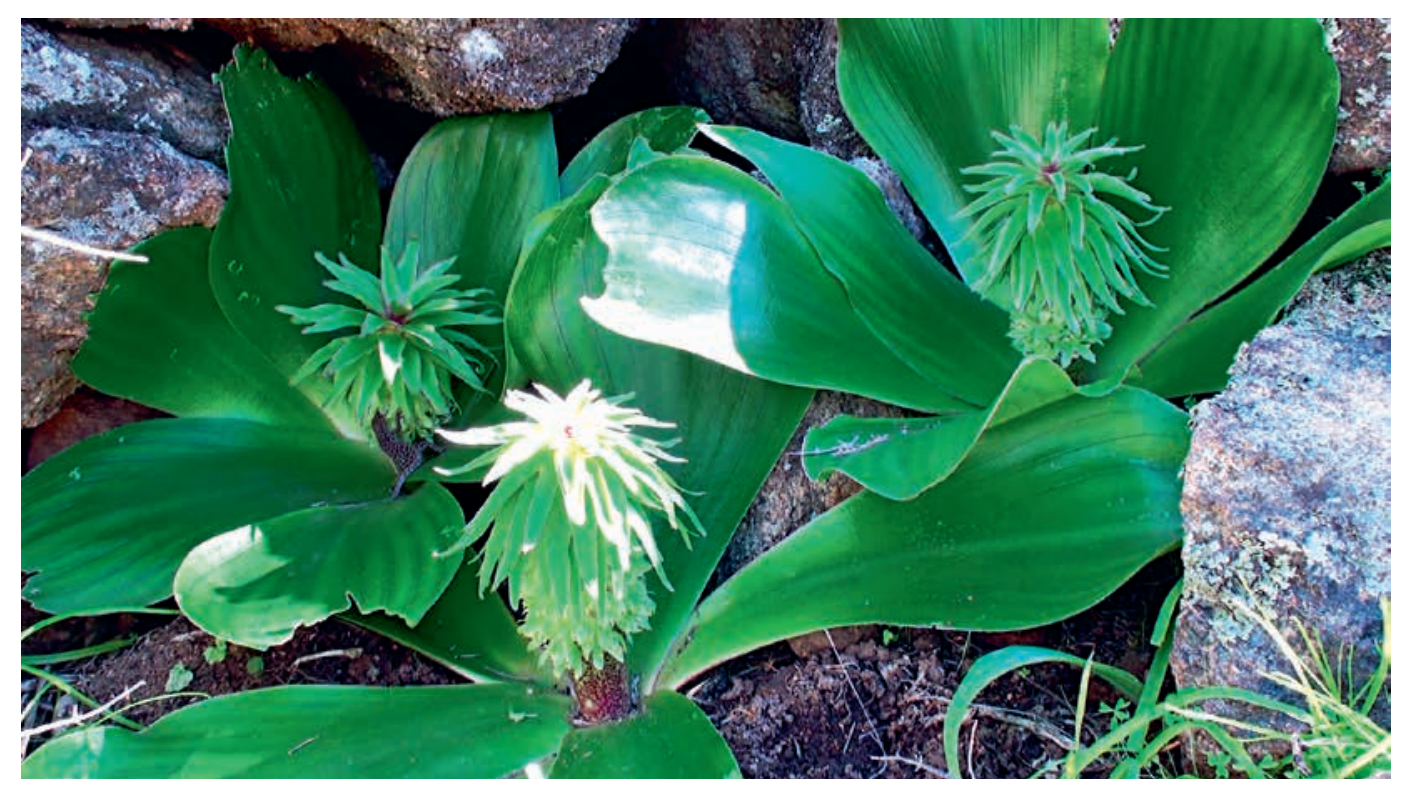

Abb. 2: Drei Ananaslilien (Eucomis regia) im Namaqualand. Die linke Pflanze wird von einem Namaqua-Taggecko (Rhoptropella ocellata) besucht. (Foto: aus Videomaterial von P. WESTER) 


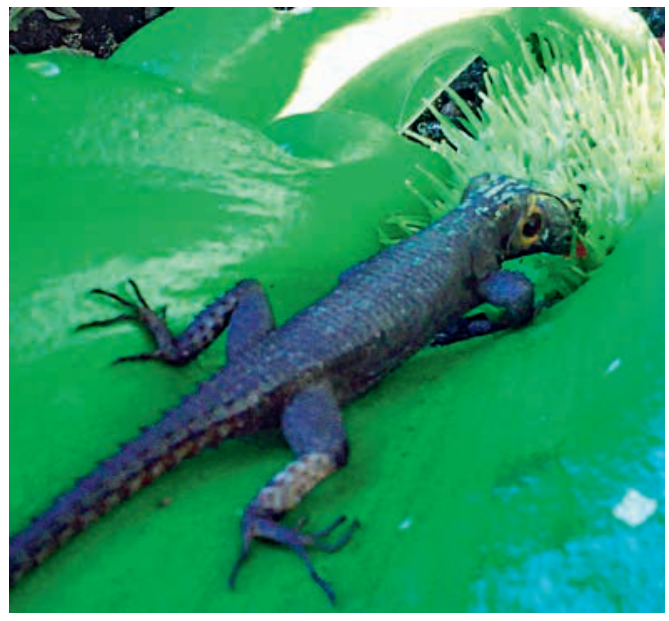

Abb. 3: Ein anderer Kap-Gürtelschweif besucht Massonia grandiflora-Blüten, leckt Nektar mit seiner rosa Zunge und berührt dabei die Antheren und Narben der Blüten. (Foto: aus Videomaterial von P. Wester)

Narben der Blüten in Berührung kamen, ist es sehr wahrscheinlich, dass Echsen zur Bestäubung der Pflanzen beitragen. Nichtsdestotrotz muss die Bedeutung der Eidechsen als Bestäuber detaillierter mit Beobachtungen und Experimenten untersucht werden. Dies könnte die Übertragung des Pollens auf die Narben zeigen oder erfolgreichen Samenansatz nach Bestäubung.

Diese Beobachtungen von zwei Eidechsenarten als Nektarkonsumenten in Südafrika sind sehr bemerkenswert, da Echsen vorwiegend Invertebraten (vor allem Arthropoden wie Insekten und Spinnen) verzehren, aber auch Pflanzenteile wie Früchte, Samen und Blätter (Schwenk 2000). Nektarkonsum durch Echsen beobachtet man nur selten. Während Ausbreitung von Samen durch Echsen schon seit langer Zeit bekannt ist, ist die Kenntnis von blütenbesuchenden und bestäubenden Echsen relativ neu (Elvers 1977, Olesen \& Valido 2003).

\section{Neue Beobachtung für das afrikanische Festland}

Interessanterweise findet man blütenbesuchende, nektartrinkende oder bestäubende Eidechsen fast ausschließlich auf Inseln von Afrika, Amerika und Australien/Ozeanien (Olesen \& VAlido 2003).
Die vorliegenden Beobachtungen zeigen erstmalig, dass Echsen auch auf dem Festland von Afrika Blüten besuchen und Nektar trinken. Der Namaqua-Taggecko ist auf das Namaqualand Südafrikas beschränkt und ernährt sich normalerweise nur von Arthropoden. Andererseits gibt es Arten der nah verwandten Gattung Phelsuma (Phelsuma ocellata ist ein Synonym von Rhoptropella ocellata), die auf Inseln als Blütenbesucher und Bestäuber agieren (Hansen \& Müller 2009, Le Péchon et al. 2013). Zusammen mit Vertretern der Geckos (Gekkonidae) sind einige Arten von sechs anderen Eidechsen-Familien als Nektarkonsumenten oder Bestäuber bekannt. Mit dem Kap-Gürtelschweif, der endemisch für Südafrika ist (vor allem im Westkap), kann eine achte Echsenfamilie (Cordylidae, Gürtelschweife) diese Liste ergänzen.

\section{Literatur}

Elvers, I. 1977: Flower-visiting lizards on Madeira. - Bot. Not. 130: 231-234.

Hansen, D. M. \& Müller, C. B. 2009: Reproductive ecology of the endangered enigmatic Mauritian Roussea simplex (Rousseaceae). - Int. J. Plant Sci. 170: 42-52.

Le Péchon, T., Sanchez, M., Humeau, L., Gigord, L. D. B. \& ZHANG L.-B. 2013: Vertebrate pollination of the endemic Trochetia granulata (Malvaceae) on Réunion. - J. Trop. Ecol. 29: 353-356.

Olesen, J. M. \& Valido, A. 2003: Lizards as pollinators and seed dispersers: an island phenomenon. - TREE 18: 177-181.

Schwenk, K. 2000: Feeding in lepidosaurs. pp. 175-291 in: Schwenk, K. (Hrsg.) Feeding: form, function and evolution in tetrapod vertebrates. - San Diego.

Wester, P. 2009. Von Mäusen und Pavianschuhen - Erste Geländebeobachtungen von Nager-Bestäubung in Südafrika. Palmengarten 73: 120-125.

Wester, P. 2011: Klebrige Nascherei für Rüsselspringer: Elephantulus edwardii als Bestäuber von Whiteheadia bifolia. Palmengarten 75: 122-125.

Wester, P., Johnson, S. D. \& Pauw, A. 2019. Scent chemistry is key in the evolutionary transition between insect and mammal pollination in African pineapple lilies. - New Phytol. 222: $1624-1637$.

\section{Anschrift der Autorin}

Dr. Petra Wester, School of Life Sciences, University of KwaZulu-Natal, P.B. X01, Pietermaritzburg 3209, Südafrika, E-Mail: westerpetra3@gmail.com 